\title{
A Force-Isotropic Underactuated Finger
}

\author{
Sébastien $\mathrm{Krut}^{\dagger}$ \\ LIRMM, UMR 5506 CNRS-UM2 \\ 161 rue Ada, 34392 MONTPELLIER CEDEX 5, FRANCE \\ krut@lirmm.fr
}

\begin{abstract}
This paper presents the design of a new underactuated finger. The main characteristic of this twodegree-of-freedom / one-actuator finger is to be force-isotropic. This means that the intensity of the forces exerted at the center of each phalanx, on the grasped object, are identical. Until now, this characteristic was observed on some underactuated fingers for some very specific configurations only (mainly when the phalanxes were lined up). With the proposed finger, this characteristic remains true whatever the configuration of the phalanxes is.
\end{abstract}

Keywords: Robotic hand, underactuation, force isotropy, cam-tendon.

\section{INTRODUCTION}

Advanced robotic hands have been developed with the intent of reaching the human hand dexterity and adaptation capabilities. They have been designed in order to equip dexterous manipulators or to become prosthesis for amputee people. More recently humanoid robotics has required such hands. Many dexterous hands, having several actuators (more than six), have been developed: Utah/MIT [1], Stanford/JPL (Salisbury's) [2], Belgrade/USC [3], DLR [4], etc. Meanwhile, other researchers proposed to constrain the design towards simple control and realization while keeping most of the robotic hand functionalities [5].

The strategy for reducing the number of actuators while keeping the hand capability to adapt its shape to the grasped object (in order to increase the number of contact points) is referred as underactuation. It is a way of simplifying the control while keeping the versatile grasping capability. Section II defines more precisely the concept of underactuation in robotic hands. A new classification of underactuation is proposed, and the relying technologies are described. A new type of underactuation mechanism based on the use of cams (pulleys with a variable radius) and pulling tendons winding around them (see Fig. 1) is introduced in Section III. For underactuated hands, usually one actuator is dedicated to the opening-closure process, but no motor controls the way the forces -exerted on the grasped object- are shared among each phalanx. This repartition is subsequent to the underactuation mechanisms and must be handled at the early design stage.

Force isotropy -equal forces exerted by each phalanx on the grasped object- is often considered as an optimal for grasping because it leads to no deterioration of the grasped object due to unbalanced force repartition. Section IV recalls this concept and points out the classical assumptions made when dealing with underactuated fingers. Section V explains what mathematical constraints must be fulfilled in order to achieve force isotropy. It points out the limits of actual underactuated fingers, and shows how "cam-tendon" mechanisms can lead to fully force isotropic fingers. Section VI gives a practical resolution for this design problem. It leads to the appropriate profiles of the cams to obtain the force-isotropic finger depicted in Fig. 1. Some extensions of this work are given in Section VII, before concluding.

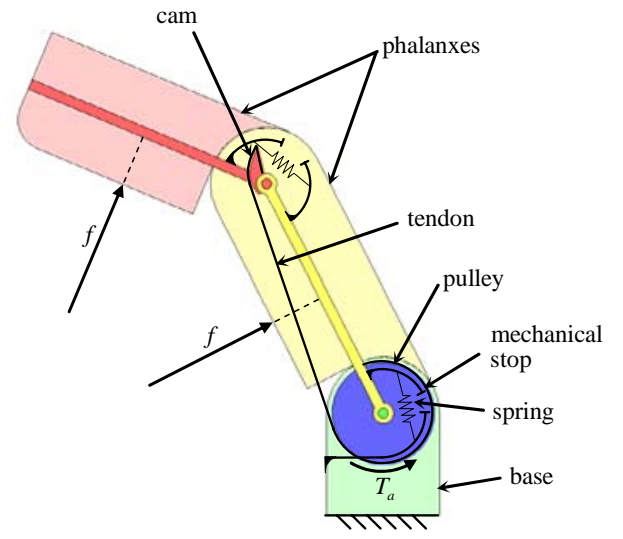

Fig. 1 - Scheme of a force-isotropic underactuated finger

\section{UNDERACTUATION IN ROBOTICS HANDS}

"A mechanism is said to be underactuated when it has fewer actuators than degrees of freedom (dof)" [6]. For example the four fingers of the TUAT/Karlsruhe Humanoid Hand [7] are said to be underactuated. In fact, those systems -serial arrangements of the three phalanxes and the palm, articulated by three revolute joints- have a global mobility of two (two dof) and one actuator only. While this definition is common with conventional underactuated robotic systems (such as the cart-pole system, or the rotating pendulum [8]), underactuation is a different concept in robotic hands [9]: for conventional robotic systems, the dynamic behavior of the system is established in order to predict unactuated joints motions, whereas for underactuated hands, the configuration of the hand is determined by the geometrical constraints associated to the grasped object - when graspingand is constrained in a particular situation (thanks to the use of springs and mechanical stops) -when not performing a grasp-.

However, it appears that the notion of dof has been extended to characterize mechanical systems where:

- Kinematic irreversibility is introduced. The gripper developed at University of Laval (Canada) for the Canadian Space Agency is said to have 10 dof [10], but the backdrivability of each finger has been removed thanks to the use of worm gears.

- Non-rigid bodies are used. The underactuated prosthetic hand proposed at the Arts Lab (Italy) [11] relies on an "adaptive grasp mechanism" designed to share the forces among each finger using compression springs.

As a conclusion, for underactuated hands, the "dof terminology" is not used as in the Grübler formula

\footnotetext{
${ }^{\dagger}$ This work has been started while being at JRL (AIST/IS-CNRS/STIC Joint Japanese-French Robotics Laboratory), Tsukuba, Japan.
} 
(representing the rank of the kinematic system) [12], but represents the number of independent parameters able to characterize all the feasible motions of the mechanism (also called "configuration variables" [13]); in other words it is the dimension of the configuration space. According to this point of view, the Barett Hand [14] is also underactuated, because each finger has two possibilities of motion but only one actuator (when considering the opening-closure process only). Hence the following categorization of underactuation mechanisms can be done:

- Differential mechanisms: they can rely on classical technologies -such has the planar differential used in the thumb of the hand proposed in [15]- or be made by specific arrangements of linkages [10] or pulleys and cables [16].

- Compliant mechanisms: non-rigid bodies are used, such as in the "adaptive grasp mechanism" proposed in [11].

- Triggered mechanisms: once the torque exceeds a certain value, the joint locks. On the Barett Hand, the transmission disengages and an irreversible mechanism prohibits backdrivability of the joint [17]. On the hand proposed by Lee, this is done, thanks to the use of automatic brakes [18].

Note: Few of the strategies depicted above can be involved simultaneously in the design of a robotic hand. For example, when considering the hand proposed in [11], a differential mechanism is used for actuating each phalanx, while a compliant device is used for the actuation of each finger. Additionally, a worm gear is used to remove backdrivability.

The scope of our study is underactuation relying on differential mechanisms. "A differential mechanism is a mechanism in which the amount of dynamical inputs from three ports acts in balance" [19]. From a kinetostatic point of view this results in:

$$
\begin{aligned}
& T_{1} / a_{1}=T_{2} / a_{2}=T_{3} / a_{3}, \\
& a_{1} \cdot \theta_{1}+a_{2} \cdot \theta_{2}+a_{3} \cdot \theta_{3}=0,
\end{aligned}
$$

where $T_{i}, i \in\{1,2,3\}$, are the torques (forces) of a three-port differential mechanism and $\theta_{i}$ the angles (displacements). Parameters $a_{i}, \quad i \in\{1,2,3\}$, describe the transmission characteristics, and may depend on the configuration of the mechanism $\left(a_{i}=a_{i}\left(\theta_{1}, \theta_{2}, \theta_{3}\right)\right)$. Next section presents a new type of differential underactuated mechanisms: "camtendon" mechanisms.

\section{III. “CAM-TENDON” UNDERACTUATED FINGERS}

\section{A. Introduction}

This section introduces a new category of underactuated devices: "cam-tendon" mechanisms. Very similar to the "pulley-cable" type, this new category relies on cams instead of pulleys. The cams can be seen as pulleys with variable radius. They are acting on tendons winding around them (not on push rods). We will see how this modification is actually of great interest, since it can lead to perfectly force-isotropic fingers. The necessary formulas for the analysis are established.

Birglen has introduced in [20] a unified approach for the kinetostatic analysis of underactuated fingers. It is based on the relationship between the input torque of the finger (generated by the actuator) and the contact forces between the grasped object and the phalanxes. This relationship involves the determination of the "transmission ratios" $R$ depending on the mechanism characteristics. The author also gives the way to compute those factors for the main types of underactuated fingers. We will recall the formulas for the "four-bar linkage" and the "pulley-cable" types, and see how we can extend them to the new "cam-tendon" type. Note: In the following development, for the sake of clarity, formulas will be given for 2-dof (degree-of-freedom) / 1actuator underactuated fingers, but can be generalized for n-dof / 1- actuator mechanisms.
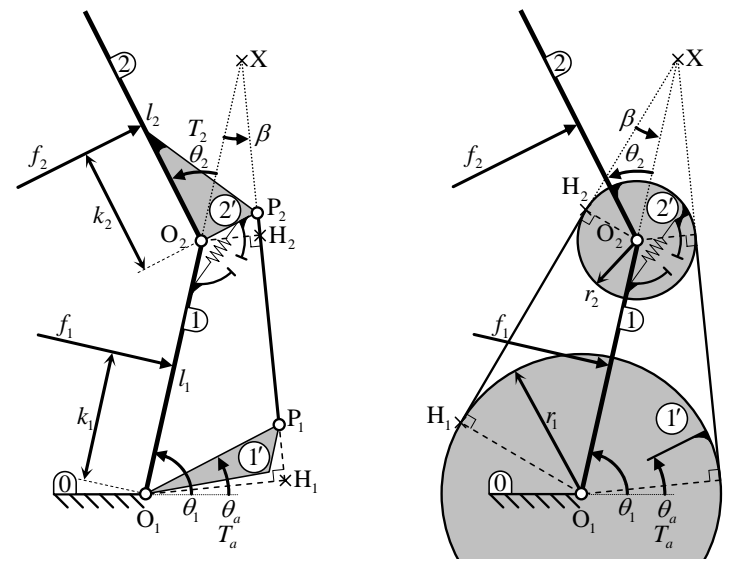

Fig. 2 - (a) "Four-bar linkage” and (b) “pulley-cable” underactuated fingers

The analytical expression of the contact forces $f_{i}$, $i \in\{1,2\}$, has been established in [9] (the contact forces are assumed to be normal and frictionless), that is:

$$
\boldsymbol{f}=\left[\begin{array}{l}
f_{1} \\
f_{2}
\end{array}\right]=\left[\begin{array}{l}
\frac{k_{2}(1+R)+R l_{1} \cos \theta_{2}}{k_{1} k_{2}} T_{a}-\frac{k_{2}+l_{1} \cos \theta_{2}}{k_{1} k_{2}} T_{2} \\
-\frac{R}{k_{2}} T_{a}+\frac{1}{k_{2}} T_{2}
\end{array}\right]
$$

where $T_{a}$ is the actuation torque and $T_{2}$, the spring induced torque. $k_{i}, i \in\{1,2\}$ define the contact locations, $l_{i}$ are the lengths of the phalanxes and $\theta_{2}$ the angle between them. This expression results from a kinetostatic analysis.

Note: $\theta_{1}$ is the angle between phalanx 1 and the frame. It characterizes the global orientation of the finger but has no influence on $f_{1}$ and $f_{2}$.

Additionally, the transmission ratio is:

$\begin{cases}R=-h /\left(h+l_{1}\right) & \text { for a "four-bar linkage” transmission } \\ R=-r_{2} / r_{1} & \text { for a "pulley-cable” transmission }\end{cases}$

where $h$ is the distance between point $\mathrm{O}_{2}$ and $\mathrm{X}$ the geometric intersection of lines $\left(\mathrm{O}_{1} \mathrm{O}_{2}\right)$ and $\left(\mathrm{P}_{1} \mathrm{P}_{2}\right)$ (see Fig. 2.a). $r_{1}$ and $r_{2}$ are the radius of pulleys $1^{\prime}$ and $2^{\prime}$ (on Fig. 2.b).

Note: Since 2 is the last phalanx, part $2^{\prime}$ is rigidly connected to it. This is true for both mechanisms.

B. Another formulation of the transmission ratio for "fourbar linkage" devices

Let us now introduce on Fig. 2.a the geometrical points $\mathrm{H}_{1}$ and $\mathrm{H}_{2}$, respectively the orthogonal projections of points $\mathrm{O}_{1}$ and $\mathrm{O}_{2}$ on line $\left(\mathrm{P}_{1} \mathrm{P}_{2}\right)$, and call $r_{1}$ and $r_{2}$, respectively the distances from $\mathrm{O}_{1}$ to $\mathrm{H}_{1}$ and from $\mathrm{O}_{2}$ to $\mathrm{H}_{2}$. Since lines $\left(\mathrm{O}_{1} \mathrm{H}_{1}\right)$ and $\left(\mathrm{O}_{2} \mathrm{H}_{2}\right)$ are parallel, and 
applying the Thales' theorem on triangle $\left(\mathrm{XO}_{1} \mathrm{H}_{1}\right)$, the following equation is derived:

$$
r_{2} / r_{1}=h /\left(h+l_{1}\right) \text {. }
$$

Equation (5) leads to another formulation of the transmission ratio $R$ for "four-bar linkage" devices:

$$
R=-r_{2} / r_{1}
$$

where $r_{1}$ and $r_{2}$ are defined above.

\section{Another category of underactuated mechanisms where} the expression of $R$ still holds

If we now have a look to Fig. 2.b, one can observe the exact same triangle $\left(\mathrm{XO}_{1} \mathrm{H}_{1}\right)$. This figure corresponds to the case where:

$$
\mathrm{P}_{1}=\mathrm{H}_{1} \text { and } \mathrm{P}_{2}=\mathrm{H}_{2} \text {, }
$$

i.e. where the contact points $\mathrm{P}_{1}$ and $\mathrm{P}_{2}$ between the pulleys and the cable are the orthogonal projection of points $\mathrm{O}_{1}$ and $\mathrm{O}_{2}$-the centers of the pulleys- on line $\left(\mathrm{P}_{1} \mathrm{P}_{2}\right)$. For this mechanism the transmission ratio $R$ is also equal to $-r_{2} / r_{1}$ (see equation (4)).

Note: For a "pulley-cable" mechanism, since there is a symmetry according to line $\left(\mathrm{O}_{1} \mathrm{O}_{2}\right)$, it is identical to consider the left part (where the cable "pulls") or the right part (where the cable "pushes") of the transmission device.

As a consequence of transmission ratios being identical for both devices, we can conclude that:

- $R$ is only depending on distances $r_{1}$ and $r_{2}$ (where $r_{1}$ [respectively $r_{2}$ ] is the distance between $\mathrm{O}_{1}$ and $\mathrm{H}_{1}$ [between $\mathrm{O}_{2}$ and $\mathrm{H}_{2}$ ]),

- $R$ does not depend on the position of points $\mathrm{P}_{1}$ and $\mathrm{P}_{2}$ on line $\left(\mathrm{H}_{1} \mathrm{H}_{2}\right)$.

This leads to a third type of underactuated device, where exactly the same formulas (3) hold to compute the contact forces $f_{i}$, and to establish the transmission ratio $R$ (6): the “cam-tendon” mechanisms (see Fig. 3).

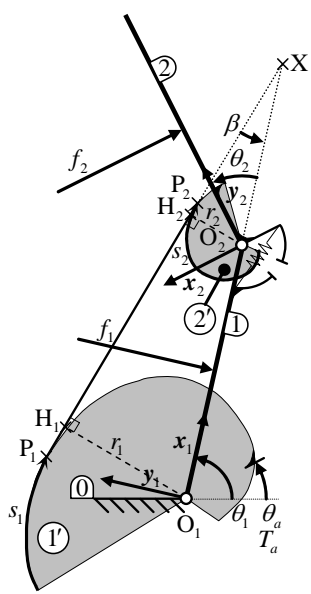

Fig. 3 - Basic shape of a "cam-tendon" underactuated finger

For this mechanism, the expression of the transmission ratio $R$ is the same as for the previous devices (see equation (6)) and relies on lengths $r_{1}$ and $r_{2}$. Note that $r_{1}$ and $r_{2}$ are not the "local" radius, but the distances from $\mathrm{O}_{1}$ to $\mathrm{H}_{1}$, respectively from $\mathrm{O}_{2}$ to $\mathrm{H}_{2} \cdot \mathrm{P}_{1}$ and $\mathrm{P}_{2}$ represent the merging points between the cable and the two cams.
Note: An important property for points $\mathrm{P}_{1}$ and $\mathrm{P}_{2}$ is that they correspond to the positions where line $\left(\mathrm{H}_{1} \mathrm{H}_{2}\right)$ is tangent to the cams.

\section{Conclusion}

In this section we have demonstrated that the way to compute the transmission ratio $R$ for "four-bar linkage" and for "pulley-cable" underactuated fingers are the same. Additionally we have proposed a third type of underactuated fingers where the previous formula holds: "cam-tendon" devices. In the following sections, we will explain how this new category of underactuated fingers, might help to achieve "force isotropy", which is an important feature for grasping.

\section{FORCE ISOTROPY}

"Force isotropy is defined as the property of a finger to generate equal contact forces on all its phalanxes" [9]. Such a characteristic is very interesting for grasping because an unbalanced force distribution might result in serious damages on the grasped object. Speaking in terms of pressure, the ultimate goal for isotropy would be to have a uniform pressure all around the wrapped object [16]. For underactuated fingers, the way the grasping forces are distributed among each phalanx depends on the transmission mechanism characteristics. As a consequence, this issue must be addressed at the early stage of design. Mathematically, -for a two-phalanx finger- force isotropy results in:

$$
f_{1}=f_{2}
$$

The "Soft-Gripper" - a “tendon-pulley" underactuated gripper- proposed by Hirose [16] was designed with the aim of being force-isotropic. The work of Hirose was of great importance because it was one of the first attempts -with [21]- to formalize underactuation for grasping. Unfortunately, his design was only force-isotropic for a specific configuration [9], namely when:

$$
\left\{\begin{aligned}
\theta_{i} & =0, i>1 \\
\text { and } k_{i} & =l_{i} / 2, i>0
\end{aligned}\right.
$$

that is to say when forces are located at the middle of each phalanx, and when the finger is perfectly straight (but can be tilted relatively to the base because $\theta_{1}$ has no influence). In fact, a closer look at system (3), shows that the contact forces depend on their positions on the phalanxes, for such a type of underactuated device. Anyhow, the middle of each phalanx is $-a$ priori- the average location of the contact point between the phalanx and the grasped object. Hence, it is quite legitimate to make the assumption that the isotropic case should be obtained for forces located at the middle of each phalanx, i.e. when equation (10) is satisfied. Another important issue to mention is that the contact forces are not exactly linear regarding to the control torque $T_{a}$. In fact there is always a residual force resulting form the spring torque $T_{2}$. As a consequence, the force distribution is not totally independent of the intensity of the control torque $T_{a}$, and force isotropy may be satisfied only for a unique value of $T_{a}$. Birglen has proposed in [9] a mathematical way to get rid of this problem. It consists in introducing the "related" spring stiffness (depending on $T_{a}$ ). In this paper 
we prefer to assume that the spring torque $T_{2}$ is neglected compared to the control torque $T_{a}$. Mathematically this results in:

$$
T_{2} / T_{a} \rightarrow 0
$$

Note: This situation is realistic in most of the cases, while the aim of the springs is only to maintain the finger straight when the hand is not performing a grasp.

What we will propose in the next section is the way to design an underactuated finger perfectly isotropic from the force repartition point of view, when assuming that the contact points are located at the middle of each phalanx, and considering that the spring torque is neglected (equations (10) and (11) will be satisfied). Contrary to the "SoftGripper”, this situation will hold for every configurations of the finger (even when $\theta_{i} \neq 0, i>0$ ).

\section{TRANSMISSION CHARATERISTICS TO ACHIEVE FORCE ISOTROPY}

In this paragraph, we will give the conditions that an underactuated mechanism must satisfy to realize force isotropy. We will explain how a "cam-tendon" underactuated device can achieve this purpose.

As a first try, we did the mathematical development for only a two-phalanx finger, but we believe that the proposed concept is more general and may be used for any-numberphalanx "cam-tendon” underactuated device. This approach will be addressed in future work when designing a "humanlike" (with 3 phalanxes) underactuated finger for a prosthesis hand. Furthermore, for the sake of clarity, we decided to choose in the following development, that:

$$
l_{1}=l_{2}
$$

When developing the isotropic condition (8) $f_{1}=f_{2}$, thanks to equation (3) -and taking into account assumptions (10) and (11) - the transmission ratio $R$ may be characterized. Once simplified, the following expression is derived:

$$
R=-1 / 4 \cos ^{2} \frac{\theta_{2}}{2}
$$

That is to say, that, to be force isotropic, an underactuated finger must have its transmission ratio verifying this particular function. Furthermore, it is important to notice that this function depends of the configuration of the finger only $\left(R=R\left(\theta_{2}\right)\right)$. Fig. 4 shows the evolution of $R$ relatively to $\theta_{2}$.

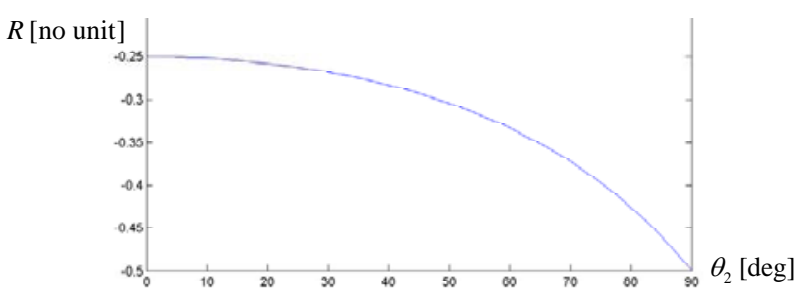

Fig. 4 - Curve $R=R\left(\theta_{2}\right)$

For "pulley-cable" mechanisms, as this factor is constant (only depending on the diameter of the pulleys) we understand that the force isotropic situation is achieved only for a particular posture of the finger (on the "Soft-Gripper", namely when $\left.\theta_{i}=0, i>1\right)$. For "four-bar linkage" mechanisms, factor $R$ depends on the lengths of the four rods, and varies according to the posture of the finger.
Birglen has exposed in [9][20] tools (mainly performance indexes) to optimize such mechanisms to become "as isotropic as possible”. Another way to run this optimization would be to minimize the distance (the absolute value of the difference) between the transmission ratio (depending on geometrical parameters) and function (13), within the whole workspace of the finger (for a set of $\theta_{2}$ ). This way, the "optimal" lengths of the four-bar transmission device would be derived. With "cam-tendon" devices we can fully satisfy this optimization problem, that is to say, we can establish the appropriate profiles of the cams to make factor $R$ satisfy statement (13) for every configurations of the finger. Let us write the mathematical relations that must be fulfilled when designing the cams to solve such a problem.

Equation (13) must be satisfied; it means that ratio $R$ defined as in equation (6) must be equal to function (13). This statement must be true for every $\theta_{2}$ corresponding to the workspace of the finger. But another relation must be also satisfied. We have to take into account that -when turning- the cams are "synchronized" by the adjacent tendon. Mathematically this can be expressed by:

$$
d s_{1}=d s_{2}
$$

where $s_{1}$ and $s_{2}$ are the arc lengths of cams $1^{\prime}$ and $2^{\prime}$ (measured from an arbitrary origin), and $d s_{i}$ designate an infinitesimal variation of distance $s_{i}$ (it is the differential of $s_{i}$ ). The computation of those values might become tricky. We will see in next section a way of getting rid of this computation. However, not computing the arc lengths holds only for 2-dof / 1-actuator underactuated fingers. This computation should be done when designing a 3-dof / 1actuator underactuated finger.

\section{PRACTICAL COMPUTATION OF THE CAMS PROFILES}

The resolution of the differential equation (14) -taking into account the isotropic condition (8)- might be complicated. However, since this differential equation needs integration, it means that a full range of solutions exists. As a consequence, instead of trying to find all solutions, we can decide to restrict the resolution to a particular solution only. This restriction should lead to major simplifications. In the following we decided to assume that:

$$
r_{1}=\text { const , }
$$

that is to say, to seek for the profile of cam $2^{\prime}$ when cam $1^{\prime}$ is a regular pulley with a constant radius. With such an assumption, $r_{2}$ becomes the only parameter of the transmission ratio $R\left(R=-r_{2} / r_{1}\right)$. With this assumption, and in the 2 dof / 1 actuator case- it becomes useless to compute the arc lengths $s_{1}$ and $s_{2}$ since it is not important to know to what extend pulley $1^{\prime}$ is tilted when $\theta_{2}$ varies.

Note: Knowing the latter relation would be useful for computing the geometrical model of the hand.

We will see in the following development how this computation falls into the determination of the envelope curve of a set of lines. The profile of the cam 2' should be described in frame $\mathfrak{R}_{2}=\left(\mathrm{O}_{2}, \mathcal{B}_{2}\right)$ (with $\mathcal{B}_{2}=\left(\boldsymbol{x}_{2}, \boldsymbol{y}_{2}\right)$, see Fig. 3) since this frame is attached to the cam. The profile can be defined as the set of points $\mathrm{P}_{2}$ for all $\theta_{2}$ in the scope $[0, \pi / 2]$, while this interval characterizes the workspace of 
the finger. (The limits of this interval were chosen arbitrarily.) Let us recall that $\mathrm{P}_{2}$ was defined as the merging point between the tendon and the cam. We already enlighten that this point corresponds to the position where line $\left(\mathrm{H}_{1} \mathrm{H}_{2}\right)$ is tangent to cam $2^{\prime}$. As a consequence, the profile of cam $2^{\prime}$ may be obtained by computing the envelope curve in frame $\mathfrak{R}_{2}$ of the set of lines $\left(\mathrm{H}_{1} \mathrm{H}_{2}\right)$ when $\theta_{2}$ increases from 0 to $\pi / 2$ (see Fig. 5).

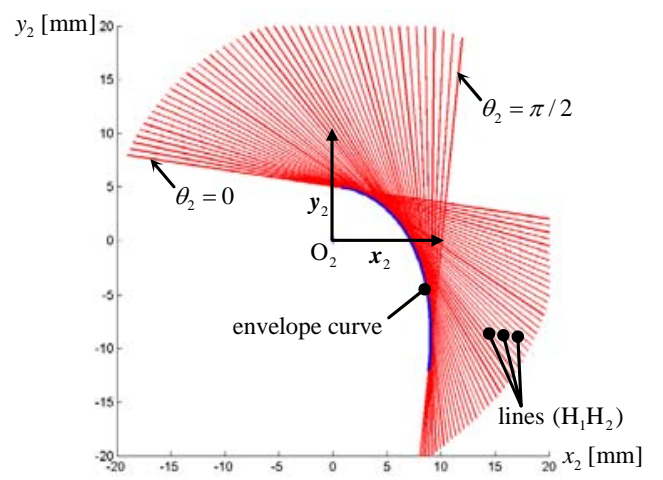

Fig. 5 - Set of lines $\left(\mathrm{H}_{1} \mathrm{H}_{2}\right)$ in frame $\mathfrak{R}_{2}$

To compute this envelope curve, let us note $\beta$, the angle between lines $\left(\mathrm{O}_{1} \mathrm{O}_{2}\right)$ and $\left(\mathrm{H}_{1} \mathrm{H}_{2}\right)$ (see Fig. 3). Taking into account the geometrical properties of points $\mathrm{O}_{1}, \mathrm{O}_{2}, \mathrm{H}_{1}$, $\mathrm{H}_{2}$ and $\mathrm{X}$ (there are flat angles between lines $\left(\mathrm{O}_{1} \mathrm{H}_{1}\right)$ and $\left(\mathrm{H}_{1} \mathrm{H}_{2}\right)$, and between $\left(\mathrm{O}_{2} \mathrm{H}_{2}\right)$ and $\left.\left(\mathrm{H}_{1} \mathrm{H}_{2}\right)\right)$ leads to:

$$
\beta=\arcsin \left(\frac{r_{1}-r_{2}}{l_{1}}\right) \text {. }
$$

Writing this expression relatively to the transmission ratio $R$ (by using relation (6)) leads to:

$$
\beta=\arcsin \frac{r_{1}}{l_{1}}(1+R)
$$

where $r_{1}$ and $l_{1}$ are geometrical constants and $R$ must satisfy equation (13) for all $\theta_{2}$.

Let us now introduce vectors $\boldsymbol{u}$ and $\boldsymbol{v}$, depending on $\beta$, described on the following scheme ( $\boldsymbol{u}$ is parallel to line $\left(\mathrm{H}_{1} \mathrm{H}_{2}\right)$, while $\boldsymbol{v}$ is orthogonal to the latter).

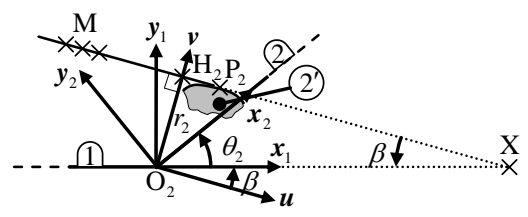

Fig. 6 - Local frames for pulley 2'

The coordinates of vectors $\boldsymbol{u}$ and $\boldsymbol{v}$ in base $\mathcal{B}_{2}$ are:

$$
\boldsymbol{u}:\left|\begin{array}{l|l}
\cos \left(\beta+\theta_{2}\right) \\
-\sin \left(\beta+\theta_{2}\right)
\end{array}, \boldsymbol{v}:{ }_{\mathcal{B}_{2}}\right| \begin{aligned}
& \sin \left(\beta+\theta_{2}\right) \\
& \cos \left(\beta+\theta_{2}\right)
\end{aligned}
$$

Thanks to those vectors, each line $\left(\mathrm{H}_{1} \mathrm{H}_{2}\right)$ might be described with the following expression:

$$
\left(\mathrm{H}_{1} \mathrm{H}_{2}\right):\left\{\mathrm{M} \mid \overrightarrow{\mathrm{O}_{2} \mathrm{M}}=r_{2} \boldsymbol{v}+u \boldsymbol{u}, u \in \mathbb{R}\right\}
$$

where $\overrightarrow{\mathrm{O}_{2} \mathrm{M}}$ represents the vector starting at $\mathrm{O}_{2}$, going to $\mathrm{M}$, and $u$ is the parameter describing the line. Line $\left(\mathrm{H}_{1} \mathrm{H}_{2}\right)$ is defined as the set of points $\mathrm{M}$, when parameter $u$ takes all real values. The coordinates of vector $\overrightarrow{\mathrm{O}_{2} \mathrm{M}}$ expressed in basis $\mathcal{B}_{2}$ are:

$$
\overrightarrow{\mathrm{O}_{2} \mathrm{M}:} \mid \begin{aligned}
& u C-r_{1} R S \\
& -u S-r_{1} R C
\end{aligned}
$$

where: $\quad C=\cos \left(\beta+\theta_{2}\right)$, and $S=\sin \left(\beta+\theta_{2}\right)$.

The envelope curve of the set of lines $\left(\mathrm{H}_{1} \mathrm{H}_{2}\right)$, when $\theta_{2}$ is in the scope $[0, \pi / 2]$, may be also characterized by equation (20), but for specific values of $u$. As a consequence the problem falls into determining $u$ for each value of $\theta_{2}$. For those specific values for $u$, points $\mathrm{M}$ become $\mathrm{P}_{2}$, the "characteristic" points of the set of lines $\left(\mathrm{H}_{1} \mathrm{H}_{2}\right)$, in other terms, the points of the lines also belonging to the envelope curve.

Thanks to Leibnitz and Taylor, the way to obtain $u$ for this envelope curve problem is known. In our specific case, it consists in solving equation:

$$
\begin{gathered}
\Delta(u)=0 \\
\text { where: } \quad \Delta=\operatorname{det}\left(\left.\left.\frac{\partial \overline{\mathrm{O}_{2} \mathrm{M}}}{\partial u}\right|_{\mathcal{B}_{2}} \frac{\partial \overline{\mathrm{O}_{2} \mathrm{M}}}{\partial \theta_{2}}\right|_{\mathcal{B}_{2}}\right) \text {. }
\end{gathered}
$$

(23) is the determinant made of the two vectors obtained when computing the partial derivative, relatively to $u$ and $\theta_{2}$, of vector $\overrightarrow{\mathrm{O}_{2} \mathrm{M}}$ when considering $\mathcal{B}_{2}$, the basis attached to cam $2^{\prime}$, as the reference (the fixed) basis. Since $\overrightarrow{\mathrm{O}_{2} \mathrm{M}}$ is expressed in basis $\mathcal{B}_{2}$ in relation (20), the partial derivatives are straightforward to obtain:

$$
\begin{aligned}
& \left.\frac{\partial \overline{\mathrm{O}_{2} \mathrm{M}}}{\partial u}\right|_{\mathcal{B}_{2}}:{ }_{\mathcal{B}_{2}} \mid-S \\
& \left.\frac{\partial \overline{\mathrm{O}_{2} \mathrm{M}}}{\partial \theta_{2}}\right|_{\mathcal{B}_{2}}: \quad \begin{array}{l}
u C^{\prime}-r_{1}(R S)^{\prime} \\
-u S^{\prime}-r_{1}(R C)^{\prime}
\end{array}
\end{aligned}
$$

where' $=\partial / \partial \theta_{2}$ represents the derivative relatively to $\theta_{2}$.

Expanding $\Delta$ leads to:

$$
\Delta=u\left(S C^{\prime}-C S^{\prime}\right)-r_{1}\left(R^{\prime}+R\left(C C^{\prime}+S S^{\prime}\right)\right)
$$

If we take into account the following relations:

$$
X^{\prime}=\frac{\partial X}{\partial\left(\theta_{2}+\beta\right)} \frac{\partial\left(\theta_{2}+\beta\right)}{\partial \theta_{2}}=\frac{\partial X}{\partial\left(\theta_{2}+\beta\right)}\left(1+\beta^{\prime}\right)
$$

where $X$ can be either function $C$ or $S\left(C^{2}+S^{2}=1\right)$,

$$
\frac{\partial C}{\partial\left(\theta_{2}+\beta\right)}=-S, \frac{\partial S}{\partial\left(\theta_{2}+\beta\right)}=C,
$$

determinant $\Delta$ falls into:

$$
\Delta=-u\left(1+\beta^{\prime}\right)-r_{1} R^{\prime}
$$

Let us point out also that:

$$
\beta^{\prime}=\frac{\partial \beta}{\partial R} R^{\prime} \text {. }
$$

Finally, solving equation $\Delta(u)=0$, leads to:

$$
u=-r_{1} R^{\prime} /\left(1+\frac{\partial \beta}{\partial R} R^{\prime}\right)
$$

where $R$ is equal to expression (13) (while it satisfies the isotropic condition (8)), and:

$$
\begin{gathered}
R^{\prime}=-\sin \frac{\theta_{2}}{2} /\left(4 \cos ^{3} \frac{\theta_{2}}{2}\right), \\
\frac{\partial \beta}{\partial R}=\frac{r_{1}}{l_{1}} / \sqrt{1-\left(\frac{r_{1}}{l_{1}}(1+R)\right)^{2}} .
\end{gathered}
$$

As a conclusion, we succeed in determining parameter $u$ as a function of $\theta_{2}$. Fig. 7 shows the evolution of 
parameter $u$ relatively to $\theta_{2}$. For numerical computations we decided to choose:

$$
l_{1}=100 \mathrm{~mm} \text { and } r_{1}=20 \mathrm{~mm} \text {. }
$$

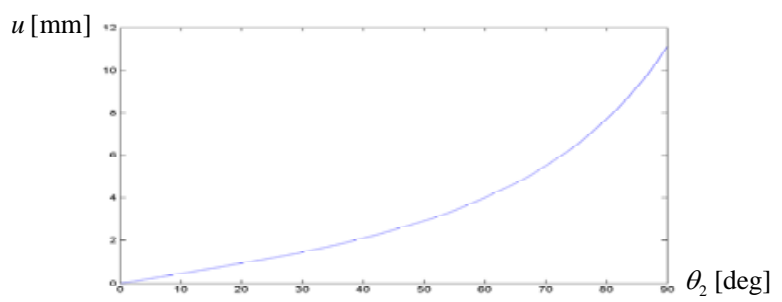

Fig. 7 - Curve $u=u\left(\theta_{2}\right)$

The characteristic points $\mathrm{P}_{2}$ of lines $\left(\mathrm{H}_{1} \mathrm{H}_{2}\right)$ are derived when redirecting parameter $u$ into relation (20). For those specific values for $u$, points $M$ become the characteristic points $\mathrm{P}_{2}$. The set of points $\mathrm{P}_{2}$, when $\theta_{2}$ takes all values between 0 and $\pi / 2$ constitutes the sought envelope curve. It is represented on Fig. 5. We can check that the obtained profile for the cam is realistic (that is to say convex) and that the tendon is able to wind around it. This envelope curve constitutes the profile of cam $2^{\prime}$. With such a cam a perfectly isotropic finger is obtained, because relation (13) is always satisfied, whatever the configuration of the finger is ( $\theta_{1} \in \mathbb{R}, \theta_{2} \in[0, \pi / 2]$ ). This finger is illustrated in Fig. 1 .

Note: The practical design of the finger requires an extra spring located between phalanx 1 and frame 0 (see Fig. 1). This is necessary when the hand opens because the tendon can only pull on the cam. The rigidity of the spring must be determined according to torque $T_{2}$, in order to obtain the desired configuration of the finger when the hand is not performing a grasp. However, the resulting spring torque was assumed to be neglected compared to the actuation torque $T_{a}$ in the above computation.

\section{FURTHER WORK}

In this paper, we explained how to compute the profiles of the cams to achieve force isotropy, but we didn't address the full analysis of the finger. For example we didn't study the ejection problem [9], or look at "fine pinch" (when not performing a "full grasp", but when only the last phalanx is involved). Furthermore, we didn't examine the sensibility of the phalanx forces relatively to errors. Errors can exist on geometrical parameters of the finger (the lengths of the phalanxes, the profiles of the cams), but also on the position of the application points of the forces while the center of each phalanx is only the "average" location of the contact points. While the proposed design is only a two-dof finger, we will extend this design to any degree of freedom. In fact, our final goal is to develop a human-like (with 3 dof per finger) prosthetic hand for amputee disabled people. The design might become complicated while the transmission factors will have to satisfy constraints -to lead to force isotropy- depending on both tilting angles (between phalanxes 1 and 2 , and phalanxes 2 and 3 ) at the same time.

\section{CONCLUSION}

In this paper, we introduced a new design of underactuated robotic hands: "cam-tendon" devices. We showed that -under some assumptions- this category of mechanism can succeed in making perfectly force-isotropic underactuated fingers. (Until now this characteristic was observed on some underactuated fingers for some very specific configurations.) We explained how to compute the profiles for the cams, and gave a practical design of a twodegree-of-freedom / one-actuator finger. We hope that this design will help making more efficient robotic hands, especially prosthetic hands for amputee people.

\section{ACKNOWLEGEMENT}

This work has been partially supported by the Japan Society for the Promotion of Science (JSPS). Also, I would like to thank warmly Dr.-Eng. Kazuhito Yokoi and Pr. Abderrahmane Kheddar, the co-directors of JRL-Japan, for welcoming me in their Laboratory.

\section{REFERENCES}

[1] S. C. Jacobsen, E. K. Iversen, D. F. Knutti, R. T. Johnson, and K. B. Biggers, "Design of the UTAH/MIT dextrous hand", in Proc. of ICRA86, San Francisco, CA, USA, 1986, pp. 1520-1532.

[2] J. K. Salisbury and J. J. Craig, “Articulated hands: Force control and kinematic issues”, The International Journal of Robotics Research, Vol. 1, No. 1, pp. 4-17, 1982.

[3] G. A. Bekey, R. Tomovic, and I. Zeljkovic, "Control Architecture for the Belgrade/USC Hand in Dextrous Robot Hands”, Springer-Verlag, NewYork, 1999.

[4] J. Butterfass, M. Grebenstein, H. Liu, and G. Hirzinger, "DLR-hand II: Next generation of a dextrous robot hand”, in Proc. of ICRA 2001, Seoul, Korea, May 21-26 2001, pp. 109-114.

[5] A. Bicchi and V. Kumar, "Robotic grasping and contact: A review," in Proc. of ICRA2000, San Francisco, CA, USA, 2000.

[6] T. Laliberté, L. Birglen and C. Gosselin, "Underactuation in robotic grasping hands,” Japanese Journal of Machine Intelligence and Robotic Control, Special Issue on Underactuated Robots, vol. 4, no. 3, pp. 77-87, September 2002.

[7] N. Fukaya, S. Toyama, T. Asfour and R. Dillmann, "Design of the TUAT/Karlsruhe Humanoid Hand”, in IROS 2000, Takamatsu, Japan, October 30 - November 5, 2000.

[8] R. Olfati-Saber, "Fixed Point Controllers and Stabilization of the CartPole System and the Rotating Pendulum”, proc. of the $38^{\text {th }}$ Conference on Decision and Control, pp. 1174-1181, Phoenix, AZ, Dec. 1999.

[9] L. Birglen and C. Gosselin, "Optimal Design of 2-Phalanx Underactuated Fingers”, in Proc. of International Conference on Intelligent Manipulation and Grasping, pp. 110-116, Genoa, Italy, July 1-2, 2004.

[10]T. Laliberté and C. Gosselin, "Actuation System for Highly Underactuated Gripping Mechanism”, United States Patent, No. US 6,505,870 B1, January 14, 2003.

[11]B. Massa, S. Roccella, M. C. Carrozza and P. Dario, "Design and Development of an Underactuated Prosthetic Hand”, in Proc. of ICRA2002, Washington, DC, May 2002.

[12]K.H. Hunt, Kinematic Geometry of Mechanisms, Clarendon Press, Oxford, 1978.

[13]R. Olfati-Saber, "Nonlinear Control of Underactuated Mechanical Systems with Application to Robotics and Aerospace Vehicles”, Ph.D. Thesis, Department of Electrical Engineering and Computer Science, Massachusetts Institute of Technology, Cambridge, MA, February 2001.

[14]W.T. Townsend, "The BarrettHand grasper - programmably flexible part handling and assembly”, Industrial Robot: An International Journal, MCB University Press, Vol. 27, No. 3, pp. 181-188, 2000.

[15]W. Zhang, Q. Chen, Z. Sun and D. Zhao, "Under-actuated passive adaptive grasp humanoid robot hand with control of grasping force", in Proc. of ICRA2003, Taipei, Taiwan, September 14-19, 2003.

[16]S. Hirose and Y. Umetani, "The development of soft gripper for the versatile robot hand”, Mechanism and Machine Theory, Vol. 13, pp. 351358, 1978.

[17]N. T. Ulrich, "Methods and Apparatus for Mechanically Intelligent Grasping”, United State Patent, No. US 4,957,320, 1988.

[18]Lee, “Artificial Dexterous Hand”, United States Patent, No. US 4,946,380, August 7, 1990.

[19]Hirose S., "Connected Differential Mechanism and its Applications”, in Proc. of ICAR85, Tokyo, Japan, September 1985.

[20]Birglen L. and Gosselin C., "Kinetostatic Analysis of Underactuated Fingers", IEEE Transactions on Robotics and Automation, Vol. 20, No. 2, pp. 211-221, April 2004.

[21]H. Shimojima, K. Yamamoto, and K. Kawakita, “A study of grippers with multiple degrees of mobility”, JSME International Journal, Vol. 30, No. 261, pp. 515-522, 1987. 